\title{
rf design of a pulse compressor with correction cavity chain for klystron-based compact linear collider
}

\author{
Ping Wang, ${ }^{1,2}$ Hao Zha, ${ }^{1,2}$ Igor Syratchev, ${ }^{3}$ Jiaru Shi, ${ }^{1,2, *}$ and Huaibi Chen ${ }^{1,2}$ \\ ${ }^{1}$ Department of Engineering Physics, Tsinghua University, Beijing 100084, People's Republic of China \\ ${ }^{2}$ Key Laboratory of Particle and Radiation Imaging of Ministry of Education, \\ Tsinghua University, Beijing 100084, People's Republic Of China \\ ${ }^{3}$ The European Organization for Nuclear Research, Geneva CH-1211, Switzerland
}

(Received 11 May 2017; published 3 November 2017)

\begin{abstract}
We present an X-band high-power pulse compression system for a klystron-based compact linear collider. In this system design, one rf power unit comprises two klystrons, a correction cavity chain, and two SLAC Energy Doubler (SLED)-type X-band pulse compressors (SLEDX). An rf pulse passes the correction cavity chain, by which the pulse shape is modified. The rf pulse is then equally split into two ways, each deploying a SLEDX to compress the rf power. Each SLEDX produces a short pulse with a length of $244 \mathrm{~ns}$ and a peak power of $217 \mathrm{MW}$ to power four accelerating structures. With the help of phaseto-amplitude modulation, the pulse has a dedicated shape to compensate for the beam loading effect in accelerating structures. The layout of this system and the rf design and parameters of the new pulse compressor are described in this work.
\end{abstract}

DOI: 10.1103/PhysRevAccelBeams.20.112001

\section{INTRODUCTION}

Pulse compressors are significantly important to linear colliders, free electron lasers (FEL), and other large facilities that use normal-conducting high-gradient accelerating structures. Klystrons, which generate long rf pulses, are widely used as power sources in accelerator facilities [1]. However, normal-conducting accelerating structures are usually operated at a low duty cycles and demand a short pulse length. The rf pulse compressor, which compresses the long rf pulse to a shorter one but with a higher peak power, meets the need in this case to reduce the investment on rf power sources of such accelerator projects as mentioned above. Pulse compressors have been in operation for many decades in many facilities, such as SLAC, Linac Coherent Light Source, SwissFEL, SPring-8 Angstrom compact free-electron laser, and PAL-XFEL [2-5]. In the blueprint of linear colliders, such as VLEPP, Next Linear Collider and Japan Linear Collider, the pulse compressors are indispensable parts of their rf units [6-8]. Moreover, in many high-power test facilities, the pulse compressor is usually the key component to enhance the test ability [9-12].

Many kinds of pulse compressors exist and are mainly classified as passive and active pulse compressors. Passive compressors include SLAC Energy Doubler (SLED),

\footnotetext{
*shij@tsinghua.edu.cn
}

Published by the American Physical Society under the terms of the Creative Commons Attribution 4.0 International license. Further distribution of this work must maintain attribution to the author(s) and the published article's title, journal citation, and DOI.
Barrel Open Cavity (BOC), and resonant delay line pulse compressor (SLED-II) [13-15]. SLED and BOC generate output pulses with an unflattened shape, which is only suitable for single-bunch acceleration, like VLEPP and SLC [16,17]. For multibunch acceleration, SLED-II is preferred because of its high efficiency and flat output pulse $[7,15,18,19]$. In principle, active pulse compressors can achieve high power gains and high efficiency [20]. Some active elements have been designed including ferroelectric, laser-driven semiconductor, plasma, electron beam injection, and multipactor switches [21-26].

Recently, the Compact Linear Collider (CLIC) updated its baseline designs at all energy stages: $380 \mathrm{GeV}, 1.5 \mathrm{TeV}$, and $3 \mathrm{TeV}$ [27]. The CLIC adopted the two-beam acceleration scheme to save on $\mathrm{rf}$ power source costs. The drive beam system of CLIC uses two linacs and a sophisticated beam-combining system that cannot be altered from stage to stage. Thus, if the two-beam acceleration scheme is applied in the $380 \mathrm{GeV}$ stage in CLIC, the drive beam system must be the same as in the later stages, and this is relatively expensive. To reduce the entry cost of CLIC, an alternative scheme using commercial X-band klystrons in the $380 \mathrm{GeV}$ stage was proposed. In this klystron-based scheme, the pulse compression is necessary to reduce the amount the klystrons used. The advantage of the scheme is that the rf units with the accelerating structures and pulse compression system can easily test the same configuration similar to that in the main linac using the drive-beam scheme at its full specifications. Two new normalconducting accelerating structures, named " $\mathrm{K}$ " and "K244," were designed for this klystron-based CLIC [27]. The accelerating structure design $\mathrm{K}$ is the cheapest 
klystron-based option. This accelerating structure is powered by an rf pulse with a length of $325 \mathrm{~ns}$ and power of $42.5 \mathrm{MW}$. Moreover, K244 is the cheapest design powered by klystrons with an rf pulse length fixed at $244 \mathrm{~ns}$. The power needed by this accelerating structure is relatively large at 54.3 MW.

Among the pulse compressors mentioned above, SLEDII pulse compressor demonstrated at SLAC is suitable for the pulse compression of the klystron-based CLIC and can be scaled to match the CLIC accelerating structures. The existing SLED-II pulse compressor deploys a dual-mode superhybrid and two delay lines, which double the delay time for a line of given length and make the rf system compact [7]. Moreover, the usage of the delay lines allows the generation of the flat output pulse, which is required by multibeam acceleration. Such a pulse compressor was considered for the $500 \mathrm{GeV}$ design of CLIC. One delay line length is $17.7 \mathrm{~m}$ and matches the pulse length of $244 \mathrm{~ns}$ for the CLIC-G accelerating structure [28,29]. Another method based on SLED or BOC, which can generate the flat pulse for the multibeam acceleration, is phase-to-amplitude modulation. In this method, a $3 \mathrm{~dB}$ hybrid combines the rf powers from two klystrons. Modulating the phases of the two klystrons results in the amplitude modulation of the output pulse with a constant phase. This procedure can remove the sharp part of the output pulse from SLED or BOC and generate a flat pulse [30]. However, the efficiency of the phase-to-amplitude method is $20 \%-25 \%$ lower than that of the SLED-II pulse compressor.

We designed a new pulse compressor for klystron-based CLIC using the accelerating structure design K244 mentioned above. This pulse compressor consists of a singlestorage cavity and a correction cavity chain. This device is extremely compact compared with the SLED-II pulse compressor. Moreover, this device can produce a flat output pulse for the multibeam acceleration with the help of phaseto-amplitude modulation using two klystrons. The efficiency of the new pulse compressor is approximately $4 \%$ lower than that of the SLED-II pulse compressor.

This paper is organized into five sections. In Sec. II, we describe the theoretical analysis of pulse compressor with correction cavities and phase-to-amplitude modulation. Then, in Sec. III, we describe the rf components of the pulse compression system. Section IV discusses parameters of the pulse compressor. Then, we formulate a conclusion in Sec. V.

\section{PULSE SHAPE CORRECTION}

\section{A. Correction with cavities}

SLED-II deploys two identical resonant delay lines to store the energy of the input pulse and generate flattop pulses [see Fig. 1(b)]. As seen from the transmission spectrum of SLED-II [see Fig. 1(a)], a series of periodically distributed resonant peaks around the operating frequency are observed [15]. The frequency interval of two nearby
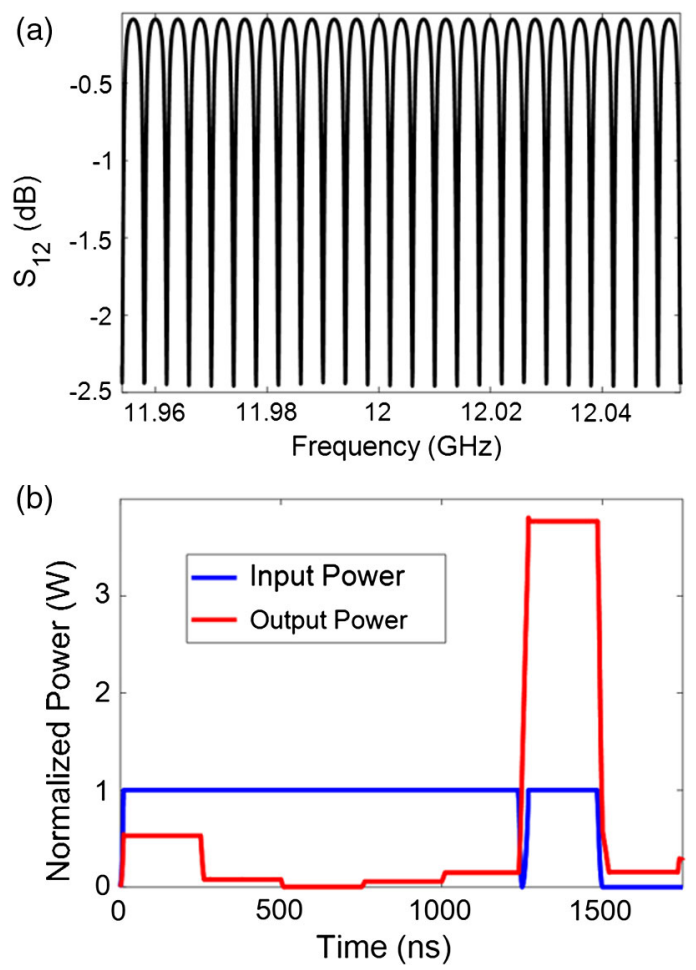

FIG. 1. (a) Center part of the transmission spectrum and (b) waveforms of SLED-II. Operation frequency is $11.994 \mathrm{GHz}$. Output pulse length is $250 \mathrm{~ns}$.

peaks is equal to the reciprocal of the round trip time of the rf pulse in the resonant delay line.

A method using several coupled cavities partially imitates the transmission spectrum of the resonant delay line as those used in SLED-II [31,32]. Each peak in the spectrum could be generated by an individual resonant cavity. The cavity for the peak at operation frequency is the storage cavity and all the cavities for other peaks are correction cavities. Figure 2 shows the scheme [Fig. 2(a)] and the network [Fig. 2(b)] for a pulse compressor with a storage cavity and several correction cavities.

In ideal conditions, the number of the correction cavities is infinite. Thus, the transmission spectra lines of the pulse compressor with correction cavities can be as dense similar to that of SLED-II. From Fig. 2(b), the relation of the input and output waves of the former $n-1$ cavities and the $n$th cavity can be described as follows:

$$
\begin{array}{r}
{\left[\begin{array}{c}
b_{1} \\
a_{1 m}
\end{array}\right]=M^{[n-1]}\left[\begin{array}{c}
a_{1} \\
b_{1 m}
\end{array}\right]=\left[\begin{array}{cc}
s_{11}^{[n-1]} & s_{12}^{[n-1]} \\
s_{21}^{[n-1]} & s_{22}^{[n-1]}
\end{array}\right]\left[\begin{array}{c}
a_{1} \\
b_{1 m}
\end{array}\right],} \\
{\left[\begin{array}{c}
b_{1 m} \\
b_{2}
\end{array}\right]=M^{n}\left[\begin{array}{c}
a_{1 m} \\
a_{2}
\end{array}\right]=\left[\begin{array}{ll}
s_{11}^{n} & s_{12}^{n} \\
s_{21}^{n} & s_{22}^{n}
\end{array}\right]\left[\begin{array}{c}
a_{1 m} \\
a_{2}
\end{array}\right] .}
\end{array}
$$

With Eqs. (1) and (2), the relation of the input and output waves of the cavity chain with $n$ cavities can be derived as follows: 
(a)
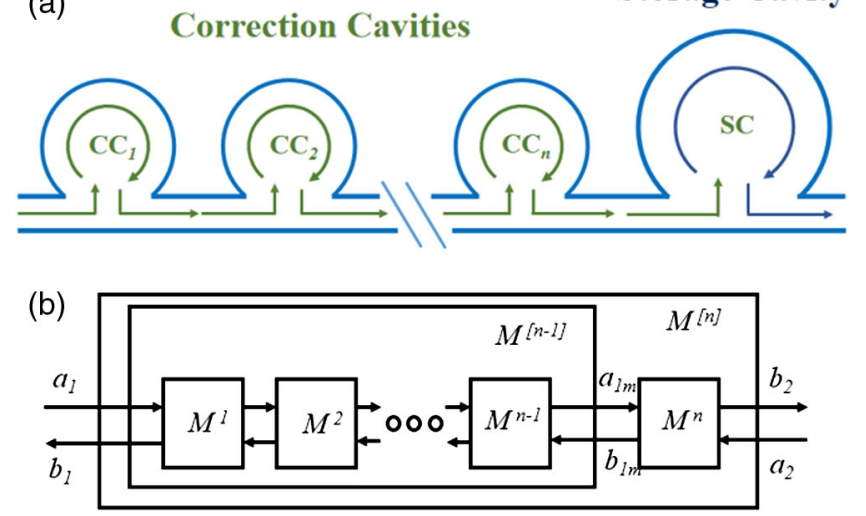

FIG. 2. (a) Scheme and (b) network of pulse compressor with a storage cavity and $n$ correction cavities. The arrows in green represent the rf power flow, which goes through the cavities one by one. The small ones are correction cavities, being of relatively low unloaded quality factor (Q-factor) and small size. The large one is the storage cavity with a large unloaded Q-factor, thereby offering the high efficiency of the pulse compression system. $M^{n}$ is the S-matrix of the $n$th cavity, and $M^{[n]}$ is the S-matrix of the cavity chain with $n$ cavities. The input waves of the $M^{[n]}$ are $a_{1}$ and $a_{2}$, and the output waves are $b_{1}$ and $b_{2}$. The input waves of the $M^{n}$ are $a_{1 m}$ and $a_{2}$, and the output waves are $b_{1 m}$ and $b_{2}$.

$$
\begin{gathered}
{\left[\begin{array}{l}
b_{1} \\
b_{2}
\end{array}\right]=M^{[n]}\left[\begin{array}{l}
a_{1} \\
a_{2}
\end{array}\right],} \\
M^{[n]}=\left[\begin{array}{cc}
s_{11}^{[n-1]}+\frac{s_{12}^{[n-1]} s_{11}^{n} s_{21}^{[n-1]}}{1-s_{22}^{[n-1} s_{11}^{n}} & \frac{s_{12}^{[n-1]} s_{12}^{n}}{1-s_{22}^{[n-1]} s_{11}^{n}} \\
\frac{s_{21}^{[n-1]} s_{21}^{n}}{1-s_{22}^{[-1]} s_{11}^{n}} & s_{22}^{n}+\frac{s_{21}^{n} s_{22}^{[n-1]} s_{12}^{n}}{1-s_{22}^{n-1]} s_{11}^{n}}
\end{array}\right] .
\end{gathered}
$$

The matrix of the cavity chain with $n$ cavities can be calculated by cascading the cavities one by one and using Eq. (4). Each cavity possesses three parameters, namely, resonant frequency, unloaded Q-factor, and coupling coefficient. With the perfect matched condition, both the $S_{11}^{\mathrm{n}}$ and $S_{22}^{\mathrm{n}}$ are zero and the transmission spectrum of the $n$th individual cavity is

$$
S_{21}^{\mathrm{n}}=\frac{\left(\beta^{n}-1\right)-j Q_{0}^{n} \xi}{\left(\beta^{n}+1\right)+j Q_{0}^{n} \xi} \delta_{p},
$$

where $\beta^{n}$ is the coupling coefficient, $Q_{0}^{n}$ is the unloaded Q-factor, $\xi=\omega / \omega_{0}-\omega_{0} / \omega$, and $\delta_{p}^{n}$ is the phase shift induced by the rf component connected to the resonant cavity. The final transmission spectrum of the pulse compressor with correction cavities is as follows:

$$
S_{21}^{\mathrm{PC}}=\prod S_{21}^{i},
$$

where $S_{21}^{i}$ is the transmission spectrum of the $i$ th resonant cavity. The bandwidth of the rf components of the pulse

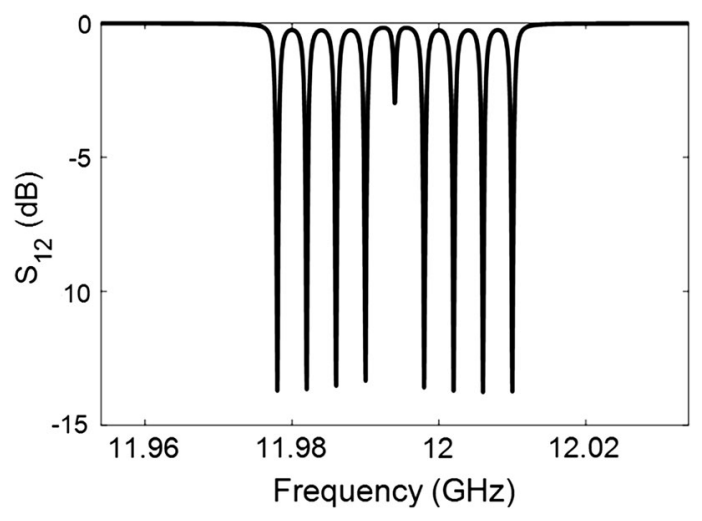

FIG. 3. Typical transmission spectrum of the pulse compressor with correction cavities. The number of the correction cavities in this case is 8 and all of them have the same unloaded Q-factor of $4.5 \times 10^{4}$ and coupling coefficient of 1.5 . Furthermore, the unloaded Q-factor of the storage cavity is $1.77 \times 10^{5}$ with a coupling coefficient of 5.98. The central frequency of the spectrum is $11.994 \mathrm{GHz}$ and the frequency difference between two nearby peaks is $4 \mathrm{MHz}$, which corresponds to the pulse length of $250 \mathrm{~ns}$.

compression system limits the number of the cavities. Therefore, the typical transmission spectrum of the pulse compressor with correction cavities has only nine peaks, as shown in Fig. 3.

An rf pulse that goes into the pulse compressor passes first through the correction cavity chain. After the rf pulse passes through the correction cavities, the shape of the pulse becomes a zigzag, as shown by the green waveform in Fig. 4(a), and the phase difference of the pulse before and after phase reversing time remains $180^{\circ}$, as shown by the blue and green waveforms in Fig. 4(b). Then, the shapemodified pulse goes into the storage cavity, which is similar to that in the SLED. The final output pulse is flat with several ripples [see red waveform in Fig. 4(a)], and the variation of the phase in the period with high peak power is within $0.02^{\circ}$, as shown in Fig. 4(c). Thus, the pulse compressor can generate such a flat pulse resulting from the similarity of the transmission spectrum of the pulse compressor with correction cavities similar to that of the resonant delay line used in SLED-II.

Given the limited number of correction cavities, several ripples are observed at the top of the output pulse. The amplitude of the ripples was reduced by increasing the number of correction cavities (see Fig. 5).

Figure 6 shows the effect of the unloaded Q-factor of the correction cavities. When the unloaded Q-factor of the correction cavities is similar to that of the storage cavity, the output pulse can be flat, as illustrated by the blue waveform in Fig. 5. However, when the unloaded quality factor is small, the shape of the output pulse is not flat but arched, as shown by the green and red waveforms in Fig. 6. The choice of low unloaded Q-factor displays the advantage of a small cavity size. Moreover, the unloaded Q-factor of the 

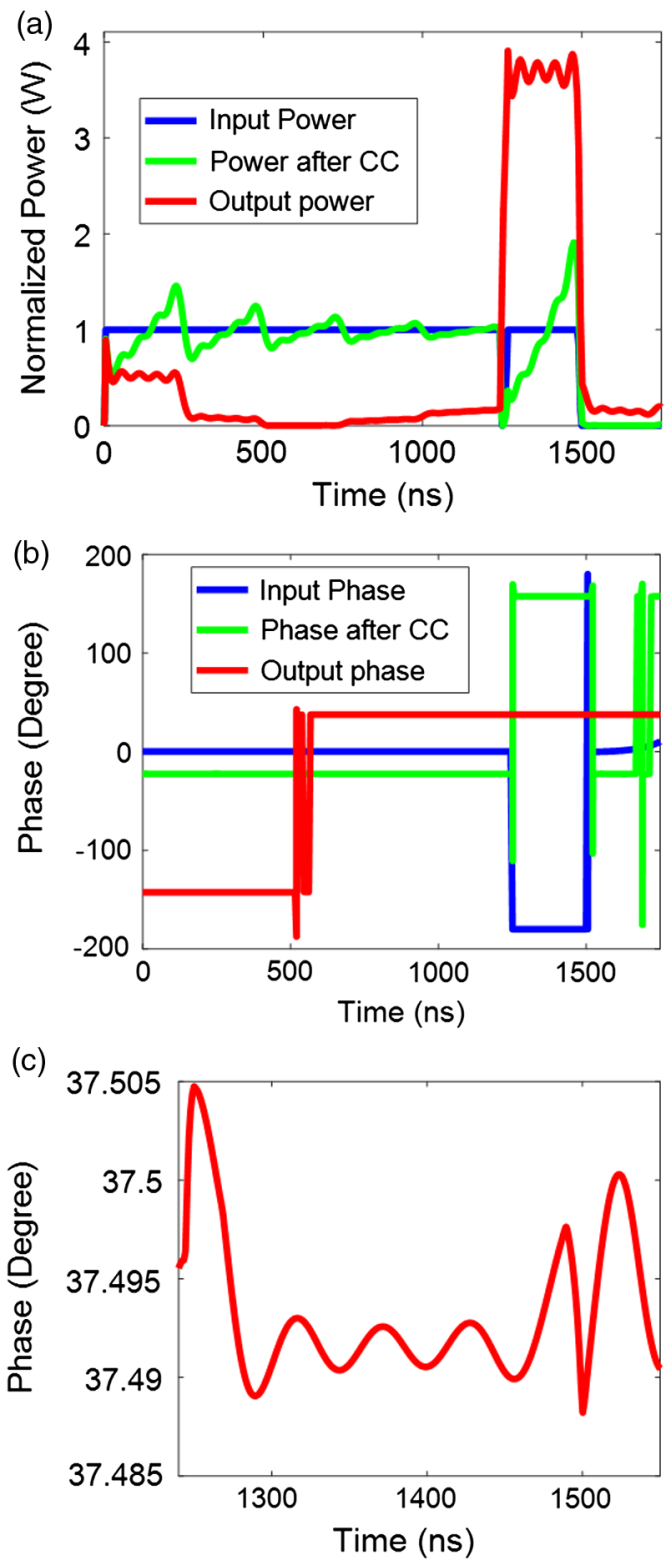

FIG. 4. (a) Input waveform, waveform after passing through the correction cavity chain, and the output waveform. (b) Input phase, phase after passing the correction cavity chain, and the output phase. (c) Output phase within the period with high peak power.

correction cavities does not have to be as large similar to that of the storage cavity. The power gain reduces by $3 \%$ (from 3.8 to 3.7) when the unloaded Q-factor is $4.5 \times 10^{4}$ compared with $1.77 \times 10^{5}$, as shown in Fig. 6. However, the unloaded quality factor should be large enough to modulate the shape of the input pulse.

\section{B. Phase-to-amplitude modulation}

The main linac of CLIC should accelerate beams with 352 bunches and the bunch separation of 5 ns in stage 1 with center-of-mass energy of $380 \mathrm{GeV}$. In this case, the input pulse for accelerating structures should have a flat

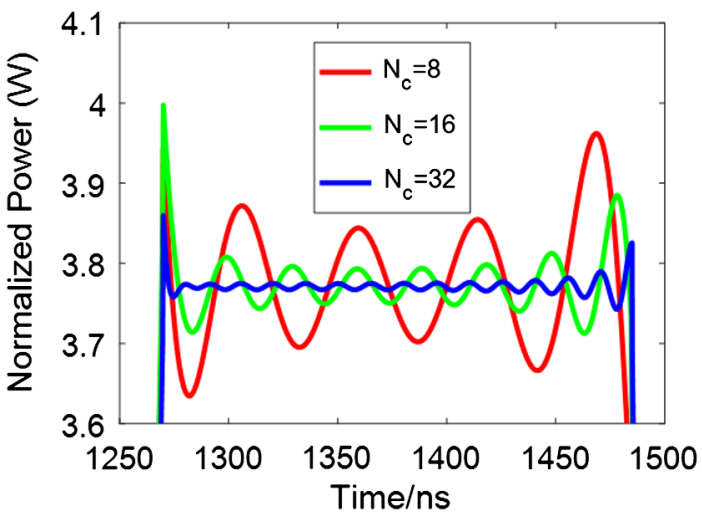

FIG. 5. Top shapes of the output pulses with different correction cavity numbers $\left(N_{c}\right)$. The output pulse length is $250 \mathrm{~ns}$ and the compression ratio is 6 . The $Q_{s}$ and $Q_{c}$ are both $1.77 \times 10^{5}$.

top. Additionally, the compensation of beam loading in the accelerating structures required that the input pulse should be of a rump-up during the filling time [33-35]. Given the limited number of correction cavities and small unloaded Q-factor, the output pulse from the pulse compressor with correction cavities cannot meet the requirement of flattop pulse and compensation of beam loading for the multibunch acceleration of CLIC. To solve this problem, the phase-to-amplitude modulation was introduced to eliminate the ripples and generate a shape of the input pulse required by beam-loading compensation. The system includes a $3 \mathrm{~dB}$ hybrid that combines the rf power from two klystrons, as shown in Fig. 7. The two klystrons can operate at the saturation condition with varying phases. The phase difference between the power from two klystrons could be adjusted so that the output power $P(t)$ could be arbitrary:

$$
\begin{aligned}
P(t) & =\left(A e^{-j[\omega t-\varphi(t)]}+A e^{-j[\omega t+\varphi(t)]}\right)^{2} \\
& =\left\{4 A^{2} \cos ^{2}[\varphi(t)]\right\} e^{-j 2 \omega t},
\end{aligned}
$$

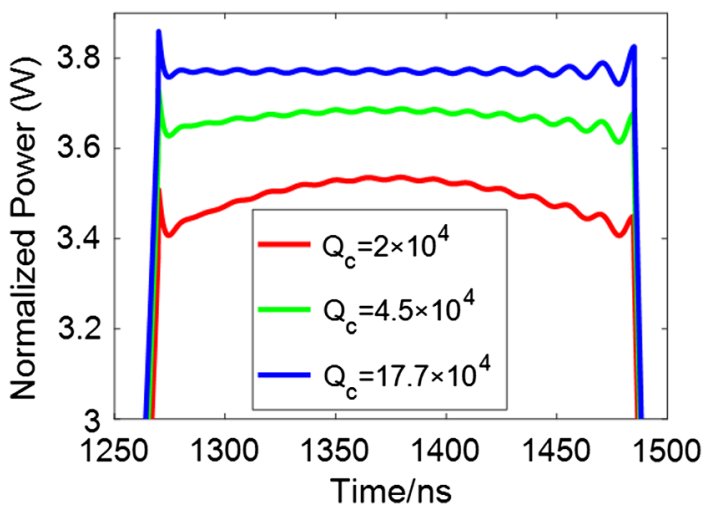

FIG. 6. Top shapes of the output pulses with different unloaded Q-factors of correction cavities. The output pulse length is $250 \mathrm{~ns}$ and the compression ratio is 6 . The $Q_{s}$ is $1.77 \times 10^{5}$ and the number of correction cavities is 32 . 


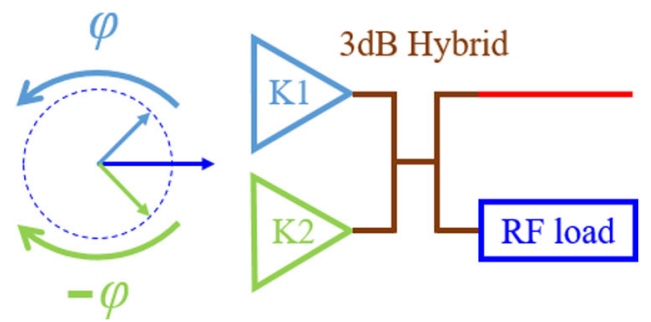

FIG. 7. Scheme of phase-to-amplitude modulation.

where $A$ is the amplitude of the field and $\varphi(t)$ is the phase to be modified. After phase-to-amplitude modulation, the shape of the end part of the input pulse was modulated to exhibit a long rising time and some ripples [see green waveform in Fig. 8(a)]. Moreover, the phases of the two klystrons are no longer constant, as shown in Fig. 8(b).

\section{III. rf DESIGN}

In this section, we describe all the rf components of the pulse compressor. A SLED-type X-band pulse compressor (SLEDX) functions as the storage cavity, which must have
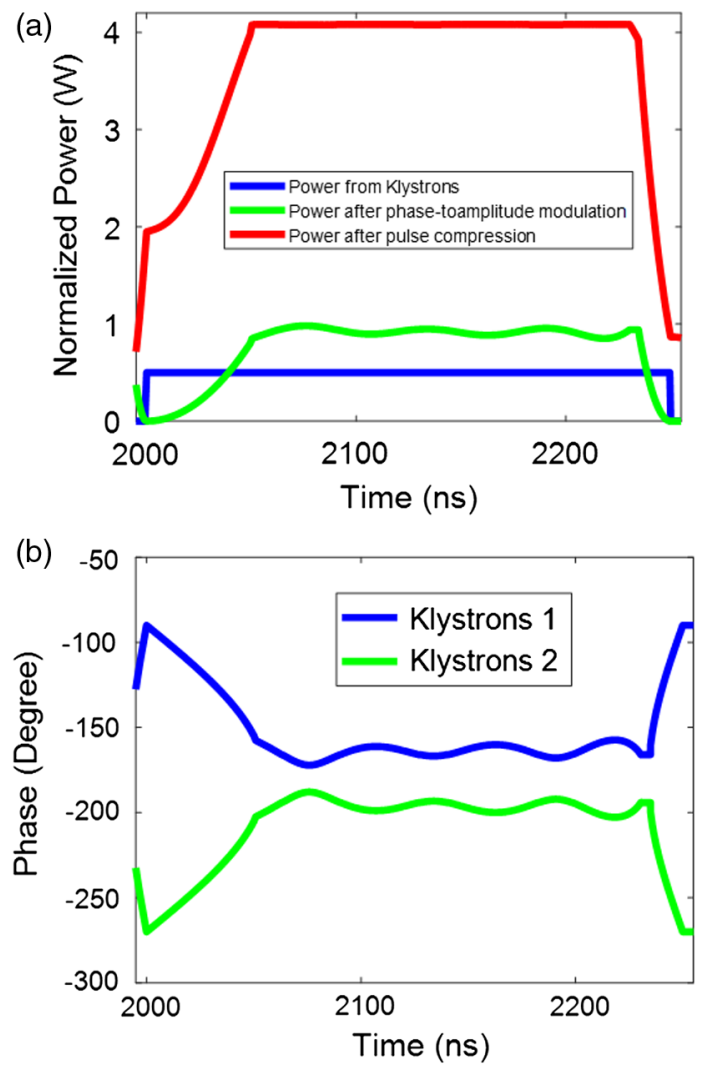

FIG. 8. (a) Waveforms of the input and output pulses after phase-to-amplitude modulation and (b) the modulated phases of the two klystrons. In this case, the compression ratio is 9 and the output pulse length is $250 \mathrm{~ns}$. The unloaded quality factors of the storage and correction cavities are $1.77 \times 10^{5}$ and $4.5 \times 10^{4}$, respectively. The number of the correction cavities is 8 . a large unloaded Q-factor. A correction cavity chain with four units modulates the shape of the input pulse. Each correction cavity unit comprises a dual-mode polarizer, two spherical cells, and a small coupling cell.

\section{A. Storage cavity}

The SLEDX is the key element in the system. Such a pulse compressor was developed at CERN [36] and has been installed in the CLIC high-gradient test facility (Xbox2) since 2014. The parameters of the SLEDX design were optimized to provide the compression from $1.5 \mu \mathrm{s}$ (generated by klystrons) to $244 \mathrm{~ns}$ pulses (required by the CLIC accelerating structure design). The storage cavities operate in the $\mathrm{H}_{0,1,32}$-mode, with a length of $45 \mathrm{~cm}$, an unloaded Q-factor of $1.77 \times 10^{5}$, and coupling coefficient of 5.98. For more details of SLEDX, see [36].

\section{B. Correction cavities}

The correction cavity chain comprises four units. Each unit contains two cavity cells, namely, a coupling cell and a dual-mode polarizer. The elected shape for the correction cavities is a spherical one, wherein the working modes are $\mathrm{TE}_{112}$, as shown in Fig. 9. Such field components are expressed as follows:

$$
\begin{gathered}
H_{r}=2 k^{2} V_{0}\left(\frac{\sin k r}{k^{3} r^{3}}-\frac{\cos k r}{k^{2} r^{2}}\right) \cos \theta \\
H_{\theta}=k^{2} V_{0}\left(\frac{\sin k r}{k^{3} r^{3}}-\frac{\cos k r}{k^{2} r^{2}}-\frac{\sin k r}{k r}\right) \sin \theta \\
E_{\varphi}=-j k^{2} \eta V_{0}\left(\frac{\sin k r}{k^{2} r^{2}}-\frac{\cos k r}{k r}\right) \sin \theta
\end{gathered}
$$

where $k$ is the wave propagation constant, $\eta$ is the impedance of free space, and $V_{0}$ is the field amplitude. Given the geometrical symmetry of the sphere, the three modes, $\mathrm{TE}_{012}$ and two degenerate $\mathrm{TE}_{112}$ modes, have the same mode patterns, except that they are rotated by $90^{\circ}$ in space from each other. The fields on the surface of the spherical cavity, compared with those in the central part, are small. This feature enables the cavity to have a large unloaded Q-factor. Given the small subscript number of the

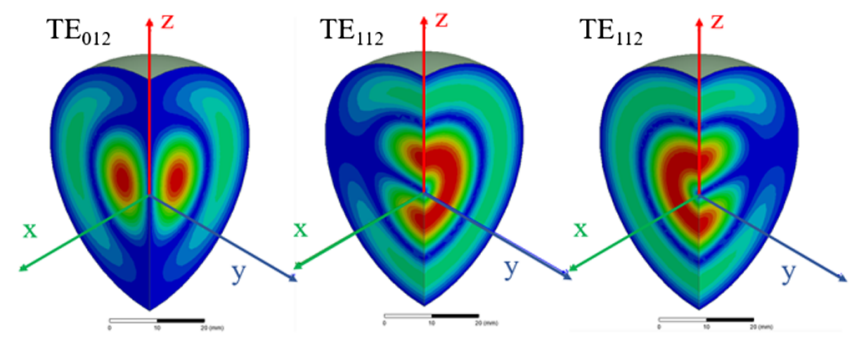

FIG. 9. Field patterns from different views of spherical resonant cavities with $\mathrm{TE}_{012}$ and two $\mathrm{TE}_{112}$ modes. 

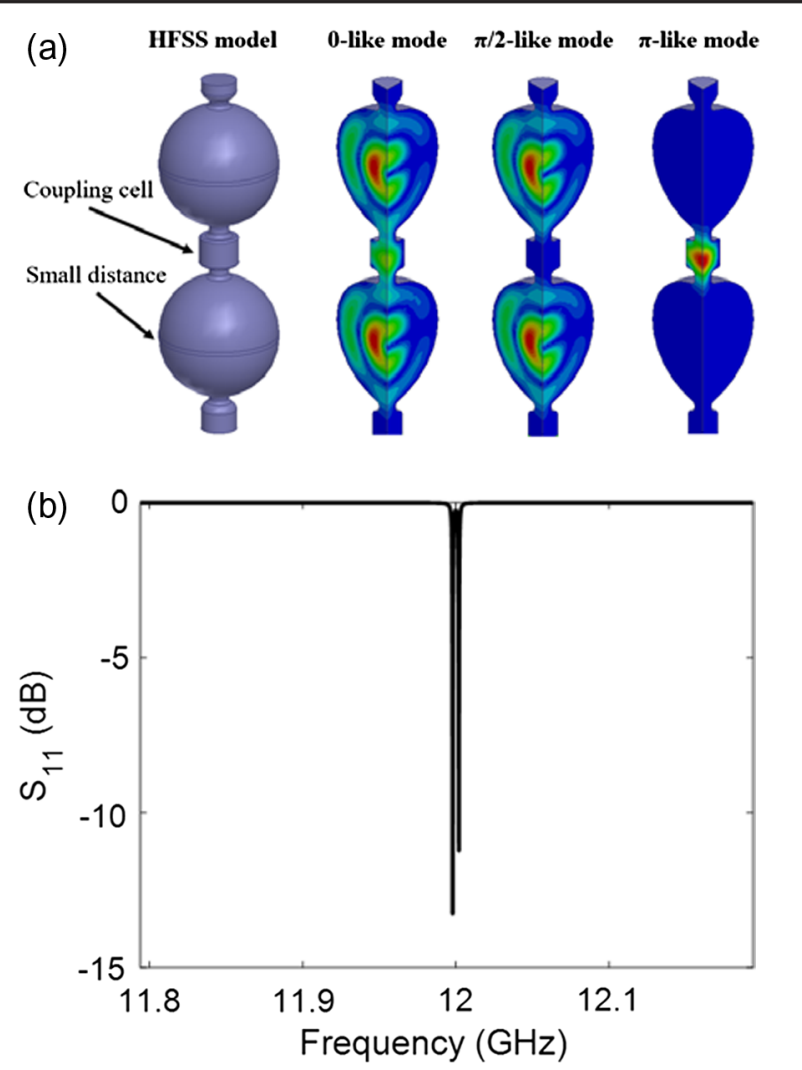

FIG. 10. (a) Field patterns and (b) reflection coefficient of a correction cavity unit.

mode, frequencies of the higher- and lower-order mode are both far from that of the operation mode.

The two cells couple with each other via a small cylinder cell, as shown in Fig. 10(a). Each cell comprises a thin (several millimeters) cylinder being sandwiched by two hemispheres. The separation of the two hemispheres tunes the cell frequency. All the spherical cells of the correction cavities are of the same radii.

The correction cavity unit with two coupled spherical cells and a small cylinder cell shows three modes of field patterns, namely, the 0 -like, the $\pi / 2$-like, and the $\pi$-like modes, as shown in Fig. 10(a). Among the three modes, the field of the $\pi$-like mode mainly distributes in the small cylinder cell, which makes the frequency more than $100 \mathrm{MHz}$ distant from the other two modes. Moreover, the field in the bottom spherical cell is small; thus, the coupling of the cavity with the waveguide is extremely weak, which causes the $\pi$-like mode to disappear in the spectrum, as shown in Fig. 10(b). The size of the coupling cell varies; thus, the field of the 0 -like mode varies, whereas the field of $\pi / 2$-like mode is unchanged. Thus, turning the geometry of the small cylinder cell can change the frequency difference between these two modes. The coupling hole between the cavity and the circular waveguide can change the external Q-factor of the two modes. The well-liked shape at the top of the correction cavity unit
TABLE I. Unloaded Q-factor and coupling coefficient of correction cavities.

\begin{tabular}{lccc}
\hline \hline$f(\mathrm{GHz})$ & $Q_{0}$ & $Q_{e}$ & $\beta$ \\
\hline 11.978 & 40537 & 29610 & 1.37 \\
11.982 & 47703 & 29753 & 1.60 \\
11.986 & 40762 & 29434 & 1.38 \\
11.990 & 47920 & 29767 & 1.61 \\
11.998 & 41015 & 29593 & 1.39 \\
12.002 & 47957 & 29626 & 1.62 \\
12.006 & 41169 & 29649 & 1.39 \\
12.010 & 47954 & 29651 & 1.62 \\
\hline \hline
\end{tabular}

keeps the geometrical symmetry to facilitate designing. The final Q-factors and coupling coefficients are shown in Table I.

\section{Dual-mode polarizer}

The dual-mode polarizer of a correction cavity unit is a three-port rf component with two rectangular ports and a circular port [37,38], as shown in Fig. 11. This polarizer was first applied to the pulse compressor by SLAC $[39,40]$. The input waveguides have regular WR90 dimensions. However, the size of the rectangular waveguide connected to the circular waveguide allows $\mathrm{TE}_{10}$ and $\mathrm{TE}_{20}$ at the working frequency. The radius of the circular waveguide and the small cylinder opposite are dimensioned so that only the $\mathrm{TE}_{11}$ mode is allowed in the propagation.

The geometry of the polarizer is carefully designed to minimize the reflection from two input ports, as well as to ensure that the orthogonality values of two $\mathrm{TE}_{11}$ modes are converted from two input ports. These $\mathrm{TE}_{11}$ modes can excite $\mathrm{TE}_{112}$ modes with different polarizations in the resonant cavity. Two polarized modes in one cavity have the same function as the two identical cavities in the SLEDI compressor. Furthermore, the dual-mode polarizer can be regarded as the $3 \mathrm{~dB}$ hybrid. Therefore, using this dualmode polarizer with the sphere cavity, the number of

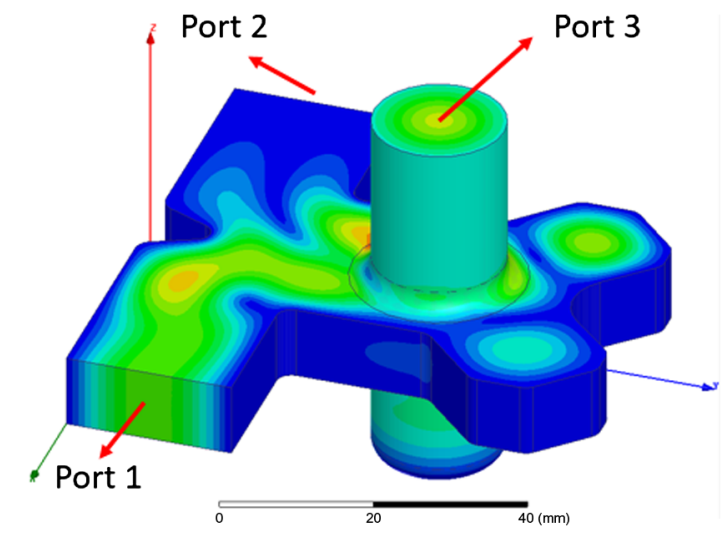

FIG. 11. Geometry and E-field distribution at $1 \mathrm{~W}$ input power injected from port 1 . 


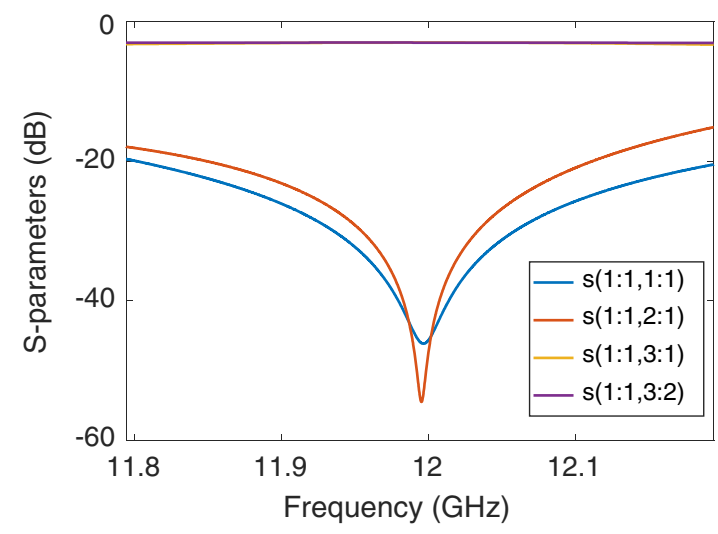

FIG. 12. S-parameters of the dual-mode polarizer.

cavities could be reduced by half. Figure 12 shows the S-parameters of the optimized polarizer. The $30 \mathrm{~dB}$ passband is $80 \mathrm{MHz}$ and the $20 \mathrm{~dB}$ passband is $270 \mathrm{MHz}$, which are sufficient for application in this work. Table II shows the S-parameters of the polarizer at working frequency. The two rectangular ports are isolated to nearly $-50 \mathrm{~dB}$ and the reflection is minimized to below $-45 \mathrm{~dB}$.

Figure 13 shows the assembly of the correction cavity units. Each cell has two cooling water channels at the top and bottom, as well as several symmetrical distributed holes for tuning. The height of a correction cavity unit is $238 \mathrm{~mm}$, and the diameter of all the cells is $38 \mathrm{~mm}$. The frequencies of the correction cavities can be tuned by changing the height of the thin cylinder being sandwiched by the two hemispheres mentioned above. The total length of the correction cavity chain is limited to $400 \mathrm{~mm}$.

The S-parameters of the correction cavity chain are shown in Fig. 14(a). By using the S-parameters of the correction cavity chain, the power after correction cavity chain and the output power can be calculated, as shown in Fig. 14(b). The results are similar to that from the ideal modal described above. The reason is that $\mathrm{S}_{11}$ and $\mathrm{S}_{22}$ are very small by a span of more than $100 \mathrm{MHz}$.

\section{CLIC PULSE COMPRESSION SYSTEM}

Figure 15 shows an rf unit for the klystron-based CLIC main linac. Two klystrons produce rf pulses, which are

TABLE II. Scattering matrix of polarizer from HFSS.

\begin{tabular}{lcccc}
\hline \hline $\mathrm{dB}(\operatorname{deg})$ & $\mathrm{S}: 1: 1$ & $\mathrm{~S}: 2: 1$ & $\mathrm{~S}: 3: 1$ & $\mathrm{~S}: 3: 2$ \\
\hline $\mathrm{S}: 1: 1$ & -49 & -53 & -3.01 & -3.01 \\
& $(149)$ & $(139)$ & $(-111)$ & $(159)$ \\
$\mathrm{S}: 2: 1$ & -53.7 & -45.9 & -3.01 & -3.01 \\
& $(139)$ & $(149)$ & $(-103)$ & $(-12.6)$ \\
$\mathrm{S}: 3: 1$ & -3.01 & -3.01 & -43 & -62.8 \\
& $(-111)$ & $(-103)$ & $(180)$ & $(-2.42)$ \\
$\mathrm{S}: 3: 2$ & -3.01 & -3.01 & -62.8 & -50.4 \\
& $(159)$ & $(-12.6)$ & $(-2.42)$ & $(-8.42)$ \\
\hline \hline
\end{tabular}

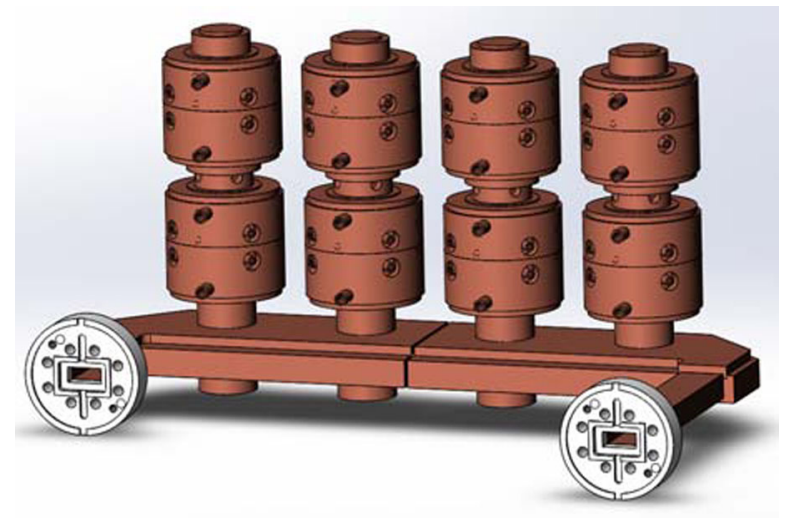

FIG. 13. Assembly of correction cavities and polarizers.

combined into a single double-power pulse. The phase-toamplitude modulation modulates the magnitude of this input pulse. Then, the pulse passes the correction cavity chain, which modifies the pulse shape, and is then split into two ways, with each sharing half the power and feeding two SLEDXs. Each SLEDX shortens the pulse by compressing the power. The utilization of two SLEDXs limits the final power in each of them, thereby stabilizing the system further. Finally, the pulses are split and distributed into
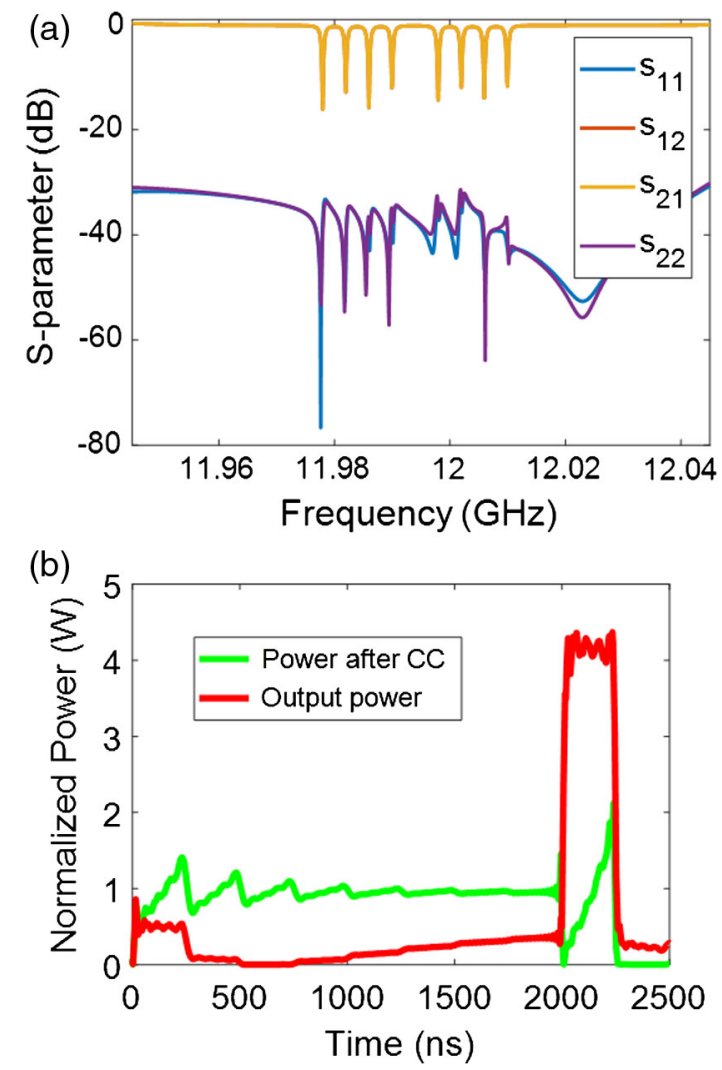

FIG. 14. (a) S-parameters of the correction cavity chain and (b) the output pulse with simulated S-parameters of correction cavity chain from HFSS and storage cavity with unloaded Q-factor of $1.77 \times 10^{5}$ and coupling coefficient of 5.98. 


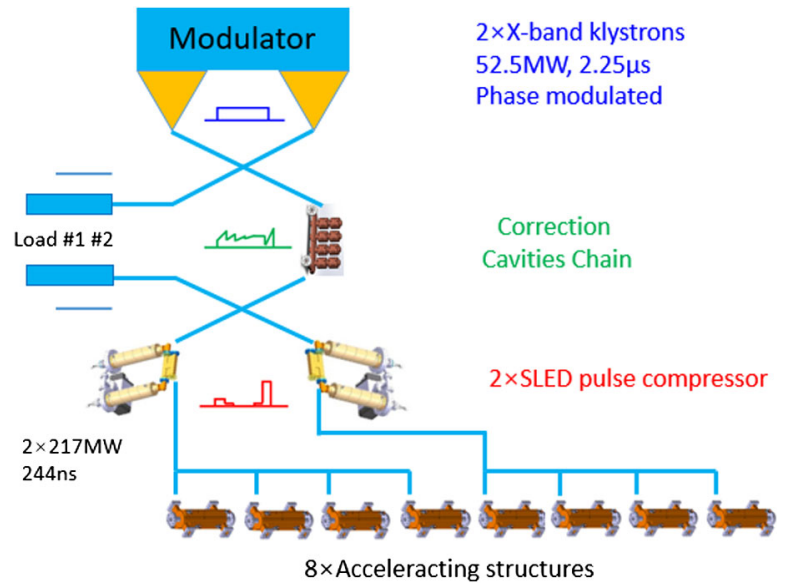

FIG. 15. Conceptual design of an rf unit for a klystron-based CLIC main linac.

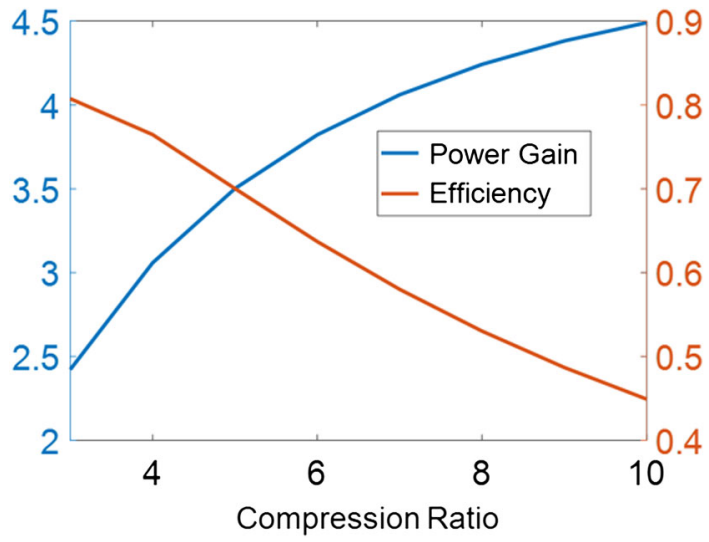

FIG. 16. Power gain and efficiency of the pulse compressor as functions of compression ratio.

eight accelerating structures. The size of the pulse compressor with correction cavity chain and even two SLEDX can be arranged in a table with a size of $1 \times 1 \mathrm{~m}^{2}$.

Figure 16 shows the power gain and efficiency of the pulse compressor with correction cavity chain as functions of compression ratio. By increasing the compression ratio, the power gain could be increased but efficiency is reduced. A long pulse length increases the power dissipated in the resonant cavities. Moreover, the structure K244 needs an input power of 54.3 MW. This power requests that the power from a klystron should be $52.5 \mathrm{MW}$ and the pulse length is $2.25 \mu \mathrm{s}$ at a compression ratio of 9 .

\section{CONCLUSION}

The rf design of the pulse compressor with correction cavities and its application to CLIC were described. With the correction cavity chain and phase-to-amplitude modulation, the SLEDX can generate the required pulse shape to compensate for the beam loading effect. The correction cavity chain and SLEDX are small and make the rf system compact, low cost, and causes it to easily provide temperature compensation and stabilization. Additionally, the pulse compressor can be easily modified to meet the pulse lengths needed by other accelerating structures.

\section{ACKNOWLEDGMENTS}

The authors are grateful to Juwen Wang and Walter Wuensch for their useful comments and to Alexej Grudiev for the discussion on the simulation of the rf polarizer. This work was supported by the National Natural Science Foundation of China (Grant No. 11375098).

[1] A. Baikov, C. Marrelli, and I. Syratchev, Toward highpower klystrons with rf power conversion efficiency on the order of 90\%, IEEE Trans. Electron Devices 62, 3406 (2015).

[2] R. Akre, V. Bharadwaj, P. Emma et al., SLAC linac rf performance for LCLS, arXiv:physics/0008171.

[3] T. Schietinger, M. Pedrozzi, M. Aiba et al., Commissioning experience and beam physics measurements at the SwissFEL Injector Test Facility, Phys. Rev. Accel. Beams 19, 100702 (2016).

[4] T. Inagaki, C. Kondo, H. Maesaka, T. Ohshima, Y. Otake, T. Sakurai, K. Shirasawa, and T. Shintake, High-gradient C-band linac for a compact $\mathrm{X}$-ray free-electron laser facility, Phys. Rev. ST Accel. Beams 17, 080702 (2014).

[5] Y. Joo, H.-S. Lee, W. Hwang, Y. Park, K. Oh, and B. J. Lee, Design study of a new SLED system with a biplanar 3-dB power divider and dual side-wall coupling-irises for the PAL XFEL, J. Korean Phys. Soc. 63, 1253 (2013).

[6] V. E. Balakin and I. V. Syrachev, Status VLEPP rf power multiplier (VPM), in Proceedings of EPAC92, Berlin, Germany (1992), pp. 1173-1175.

[7] S. G. Tantawi, C. D. Nantista, V. A. Dolgashev, C. Pearson, J. Nelson, K. Jobe, J. Chan, K. Fant, J. Frisch, and D. Atkinson, High-power multimode X-band rf pulse compression system for future linear colliders, Phys. Rev. ST Accel. Beams 8, 042002 (2005).

[8] JLC Study Group Collaboration, KEK Report No. 97-1, 1997.

[9] A. E. Vlieks, R. Callin, H. Deruyter et al., Accelerator and rf system development for NLC, in Proceedings of the 15th Particle Accelerator Conference, PAC-1993, Washington, DC, 1993 (IEEE, New York, 1993), pp. 620-622.

[10] N. Catalan-Lasheras, A. Degiovanni, S. Doebert et al., Experience operating an X-band high-power test stand at CERN, in Proceedings of the 5th International Particle Accelerator Conference (IPAC), Dresden, Germany (2014), pp. 2288-2290.

[11] N. C. Lasheras, C. Eymin et al., Commissioning of XBOX3 a very high capacity X-band rf test stand, in Proceedings, 29th Linear Accelerator Conference, LINAC 2016, East Lansing, MI, USA (2016), pp. 568-571.

[12] X. Wu, J. Shi, H. Chen, J. Shao, T. Abe, T. Higo, S. Matsumoto, and W. Wuensch, High-gradient breakdown 
studies of an X-band Compact Linear Collider prototype structure, Phys. Rev. Accel. Beams 20, 052001 (2017).

[13] Z. D. Farkas, H. A. Hogg, G. A. Loew, and P. B. Wilson, SLED: A method of doubling SLAC's energy, in Proceedings of the 9th International Conference on High Energy Accelerators (SLAC, California, USA, 1974), p. 576.

[14] I. V. Syratchev, The progress of X-band "Open" cavity rf pulse compression systems, EPAC, London, England (1994), pp. 375-379.

[15] S. Tantawi, Multimoded reflective delay lines and their application to resonant delay line $\mathrm{rf}$ pulse compression systems, Phys. Rev. ST Accel. Beams 7, 032001 (2004).

[16] V. Balakin, V. A. Sidorov, and A. N. Skrinsky, The VLEPP project status report.

[17] J. T. Seeman, The stanford linear collider, Ann. Rev. Nucl. Part. Sci. 41, 389 (1991).

[18] P. B. Wilson, Z. D. Farkas, and R. D. Ruth, SLEDII: A new method of rf pulse compression, Linear Accelerator Conference, Albuquerque, NM, USA (1990), pp. 204206; Report No. SLAC-PUB-5330, 1990.

[19] S. V. Kuzikov, A. A. Vikharev, M. E. Plotkin, D. Y. Shegol'kov, and J. L. Hirshfield, One-channel Ka-band pulse compressor, Phys. Rev. ST Accel. Beams 10, 082001 (2007).

[20] S. G. Tantawi, R. D. Ruth, A. E. Vlieks, and M. Zolotorev, Active high-power rf pulse compression using optically switched resonant delay lines, IEEE Trans. Microwave Theory Tech. 45, 8 (1997).

[21] F. Tamura and S. G. Tantawi, Development of high power $\mathrm{X}$-band semiconductor microwave switch for pulse compression systems of future linear colliders, Phys. Rev. ST Accel. Beams 5, 062001 (2002).

[22] A. L. Vikharev, High power active X-band pulse compressor using plasma switches, Phys. Rev. ST Accel. Beams 12, 062003 (2009).

[23] A. L. Vikharev, O. A. Ivanov, A. M. Gorbachev, M. A. Lobaev, V. A. Isaev, S. G. Tantawi, J. R. Lewandowski, and J. L. Hirshfield, X-band active-passive rf pulse compressor with plasma switches, Phys. Rev. ST Accel. Beams 14, 121302 (2011).

[24] O. A. Ivanov, A. A. Vikharev, A. M. Gorbachev, V. A. Isaev, M. A. Lobaev, A. L. Vikharev, S. V. Kuzikov, J. L. Hirshfield, and M. A. LaPointe, Active quasioptical Ka-band rf pulse compressor switched by a diffraction grating, Phys. Rev. ST Accel. Beams 12, 093501 (2009).

[25] O. A. Ivanov, V. A. Isaev, M. A. Lobaev, A. L. Vikharev, and J.L. Hirshfield, High power microwave switch employing electron beam triggering with application to active rf pulse compressors, Phys. Rev. ST Accel. Beams 14, 061301 (2011).
[26] O. A. Ivanov, M. A. Lobaev, A. L. Vikharev, A. M. Gorbachev, V. A. Isaev, J. L. Hirshfield, S. H. Gold, and A. K. Kinkead, Active Microwave Pulse Compressor Using an Electron-Beam Triggered Switch, Phys. Rev. Lett. 110, 115002 (2013).

[27] P. N. Burrows, P. Lebrun, L. Linssen, D. Schulte, E. Sicking, S. Stapnes, and M. A. Thomson, Report No. CERN-2016-004, 2016, http://dx.doi.org/10.5170/ CERN-2016-004.

[28] D. Schulte, A. Grudiev, Ph. Lebrun, G. McMonagle, I. Syratchev, and W. Wuensch, Report No. CERN-OPEN2013-024.

[29] D. Schulte, A. Grudiev, Ph. Lebrun, G. McMonagle, I. Syratchev, and W. Wuensch, Report No. CLIC-Note-1013.

[30] P. Wang, J. Shi, H. Zha et al., Experimental study on PMAM method in pulse compression system, in Proceedings of the 8th International Particle Accelerator Conference (IPAC), Copenhagen, Denmark, (2017), pp. 4230-4232.

[31] S. Y. Kazakov, Pulse shape correction for rf pulse compression system, at The 3rd European Particle Accelerator Conference, Berlin, Germany (1992), pp. 1247-1248.

[32] C. Xu, J. Wang, S. Tantawi, W. Juwen, S. Tantawi, and C. Xu, New SLED 3 system for multi-mega Watt $\mathrm{rf}$ compressor, arXiv:1408.4851.

[33] A. Lunin, V. Yakovlev, and A. Grudiev, Analytical solutions for transient and steady state beam loading in arbitrary traveling wave accelerating structures, Phys. Rev. ST Accel. Beams 14, 052001 (2011).

[34] O. Kononenko and A. Grudiev, Transient beam-loading model and compensation in Compact Linear Collider main linac, Phys. Rev. ST Accel. Beams 14, 111001 (2011).

[35] H. Zha and A. Grudiev, Design, and optimization of Compact Linear Collider main linac accelerating structure, Phys. Rev. Accel. Beams 19, 111003 (2016).

[36] B. Woolley, A. Dexter, I. Syratchev, and G. Burt, High power X-band rf test stand development and high power testing of the CLIC crab cavity, Ph.D. thesis, Lancaster University, 2015.

[37] M. Franzi, J. Wang, V. Dolgashev, and S. Tantawi, Compact rf polarizer and its application to pulse compression systems, Phys. Rev. Accel. Beams 19, 062002 (2016).

[38] A. Grudiev, Report No. CLIC-Note-1067.

[39] J. Wang, S. Tantawi, and X. Chen, Super-compact SLED system used in the LCLS diagnostic system, in Proceedings of the 27th Linear Accelerator Conference, LINAC 2014, Geneva, Switzerland (2014), pp. 1151-1154.

[40] J. Wang, S. Tantawi, and X. Chen et al., R\&D for a super compact SLED system at SLAC, in Proceedings of the 7th International Particle Accelerator Conference (IPAC), Busan, Korea (2016), pp. 39-41. 